\title{
Listeria monocytogenes-LLO-PSA Vaccine ADXS31-142
}

National Cancer Institute

\section{Source}

National Cancer Institute. Listeria monocytogenes-LLO-PSA Vaccine ADXS31-142. NCI

Thesaurus. Code C120103.

A cancer vaccine containing a live-attenuated strain of the Gram-positive bacterium Listeria monocytogenes $(\mathrm{Lm})$ encoding a fusion protein composed of the tumorassociated antigen (TAA) human prostate-specific antigen (PSA) fused to a fragment of the immunostimulant listeriolysin O (LLO) protein, with potential immunostimulatory and antineoplastic activities. Upon administration of the Lm-LLO-PSA vaccine ADXS31-142, the expressed LLO-PSA is processed by antigen presenting cells (APCS), presented to the immune system by both major histocompatibility complex (MHC) I and II molecules, and activates the immune system to exert both an innate and adaptive immune response involving the recruitment and activation of T-lymphocytes against PSA-expressing tumor cells as well as the inhibition of tumor-infiltrating $T$ regulatory cells (T regs) and myeloidderived suppressor cells (MDSCs). This eventually results in tumor cell lysis. 